\title{
An Improved Bayesian Decision Theoretic Approach for Land Mine Detection
}

\author{
Leslie Collins, Member, IEEE, Ping Gao, Student Member, IEEE, and Lawrence Carin, Senior Member, IEEE
}

\begin{abstract}
A rigorous signal detection theoretic analysis is used to improve detectability of land mines. The development is performed for sensors that integrate time-domain information to provide a single data point (standard metal detector), those that provide a sampled portion of the time-domain waveform, and those that operate at several discrete frequencies. This approach is compared to standard thresholding techniques, and it is shown to provide substantial improvements when evaluated on measured data.
\end{abstract}

Index Terms-Bayes procedures, data processing, electromagnetic induction, signal detection.

\section{INTRODUCTION}

$\mathbf{T}$ HE GOAL of any detection system is to achieve a high detection rate while minimizing the number of false alarms. While many mine detectors achieve the first of these goals, it is often at the cost of a prohibitively large false alarm rate. This often results from the fact that mine detectors simply seek anomalies, which may occur as a result of mines or clutter, and do not incorporate any knowledge of the statistics of the sensor response to mines and clutter. In this paper, a Bayesian decision-theoretic approach to the detection of mines is presented that incorporates the statistical properties of both the target and clutter. Results are presented for three different classes of electromagnetic induction (EMI) devices: integrated time-domain, time-domain, and frequency-domain. Detectors for each class of device are derived theoretically and evaluated based on data obtained in the field. In particular, we consider the Geonics EM61, an integrated timedomain, pulsed-induction device; the Geonics EM61-3D, a prototype, multichannel time-domain, pulsed-induction device; and Geophex's GEM-3, a prototype, broadband, frequencydomain device. Our first goal is to determine whether, at a fixed detection rate, a Bayesian decision-theoretic approach substantially reduces false alarm rates relative to the standard thresholding techniques commonly used on such data. In addition to determining whether such an approach is useful for mitigating false alarms, we investigate the degree to which additional knowledge of the time- or frequency-domain signatures (EM61-3D and GEM-3, respectively) could be used within the same Bayesian detection framework to improve per-

Manuscript received September 29, 1997; revised June 8, 1998. This work was supported by the Army Research Office under Grant DAAH04-96-1-0448 (Demining MURI).

The authors are with the Department of Electrical and Computer Engineering, Duke University, Durham, NC 27708-0291 USA (e-mail: 1collins@ee.duke.edu).

Publisher Item Identifier S 0196-2892(99)00841-4. formance over that obtained with the more standard integrated time- or frequency-domain measurements (EM61).

Using signal detection theory, the physics of each of the EMI systems is incorporated into the detector explicitly. The signal detection-theoretic approach prescribes a method for optimally processing the signals for detection and identification in an uncertain or random environment. When applied correctly, this approach guarantees optimal performance in the sense of minimum probability of error, or maximum detection rate for a given false alarm rate. Additionally, signal detection theory can be used to incorporate a priori knowledge concerning the set of values that parameters of the environment or the signal can take on. This approach provides both an improved detection algorithm and quantitative performance evaluation measures in the form of a functional relationship between probability of detection $\left(P_{d}\right)$ and probability of false alarm $\left(P_{f a}\right)$, termed the receiver operating characteristic (ROC). We present results in which the statistical, Bayesian approach significantly outperforms (lower $P_{f a}$ for the same $P_{d}$ ) the standard threshold detector on the data obtained from the DARPA backgrounds clutter data collection experiment [1], which was sponsored by the Defense Advanced Research Projects Agency.

In the remainder of this paper, the data and the sensors that were used for this analysis are described. The underlying physics of the EMI sensors is reviewed briefly and followed by a discussion of the detection strategies. In addition to describing the Bayesian detection strategies, the standard techniques commonly applied to the data are described and their relationship to the Bayesian techniques are addressed. Finally, the results of the Bayesian and standard detection techniques for each of the three sensors are discussed.

\section{REVIEW OF UNDERLYING PHYSICS}

EMI systems generally operate at very low frequencies $(<1 \mathrm{MHz})$ at which the displacement-current component to the near-field loop-induced fields can often be ignored. Therefore, such systems are relatively insensitive to dielectric discontinuities and primarily sense changes in conductivity and permeability. Thus, EMI systems are of primary use for the detection of mines with substantial metal content, or for unexploded ordnance (UXO), which generally contain significant metal. Traditional EMI systems are of little or no use for purely dielectric targets (mines).

Nevertheless, most mines have at least some metal content, and EMI systems are currently the only fieldable sensors for detecting such targets. Moreover, when operated in the time- or 
wideband frequency-domain, EMI systems afford the potential for target discrimination, which mitigates the need to dig up each piece of metal detected. In particular, it has been shown [2]-[5] that the pulsed-EMI response $s(t)$ from a general conducting, permeable target can be expressed as

$$
s(t)=\sum_{n=1}^{M} A_{n} \exp \left(-\alpha_{n} t\right) .
$$

Thus, the target response is characterized as a sum of $M$-weighted, exponentially damped modes. In practice, the lowest mode dominates the response, and only one exponential term $\left(\alpha_{1}\right)$ need be considered. Moreover, theoretical and experimental data demonstrate that $\alpha_{1}$ is unique to each metal type and is a function of conductivity and permeability. It is this phenomenon we wish to exploit to distinguish targets from clutter. The amplitude of the response $A_{1}$ corresponds to the excitation level, and it is a complicated function of, among other things, the distance between the target and sensor and the orientation of the target. In this initial work, we chose to normalize the amplitude of the response to unity to focus on the discrimination capability inherent in the decay rate, as well as to avoid uncertainty in the target to sensor distance. Thus, hereafter, $A_{1}=1$.

\section{DETECTOR DESIGN}

\section{A. Integrated Time-Domain Sensors}

In order to improve detection performance, we consider utilizing signal detection theory to incorporate the statistics of the target and clutter into the detector design. The signal model that is adopted assumes that the sensor integrates the voltage induced in the receive coil from an initial time $t_{0}$ to some final time $t_{1}$ by sampling the received signal and summing the samples to a scalar value $s$, i.e.,

$$
s=\sum_{k=0}^{N-1} \exp \left(-\alpha\left(t_{0}+k \Delta t\right)\right)
$$

where $t_{0}+(N-1) \Delta t=t_{1}$. Finally, it is assumed that the sensor is subject to additive noise $n(k)$ at each sample point so that the received data $r$ can be modeled as

$$
r=\sum_{k=0}^{N-1}\left[\exp \left(-\alpha\left(t_{0}+k \Delta t\right)\right)+n(k)\right] .
$$

1) Known Parameter Case: The detector will be formulated in terms of a binary hypothesis test assuming equally likely hypotheses. $H_{1}$ denotes the target-present hypothesis, and $H_{0}$ denotes the null, or no-target-present, hypothesis. The likelihood ratio test (LRT) to be performed on the received data $r$ is defined as

$$
\Lambda(r)=\frac{p\left(r / H_{1}\right)}{p\left(r / H_{0}\right)}
$$

where $p\left(r / H_{1}\right)$ and $p\left(r / H_{0}\right)$ are the probability density functions describing the received data under $H_{1}$ and $H_{0}$, respectively [6]. If $\Lambda(r)>\delta$, where $\delta$ is a threshold set to achieve a certain false alarm rate, $H_{1}$, is chosen, whereas, if $\Lambda(r)<\delta, H_{0}$ is chosen. If $p\left(r / H_{1}\right)$ and $p\left(r / H_{0}\right)$ are Gaussian distributions with identical variances, but different means, the likelihood ratio test reduces to the standard threshold test. However, when these assumptions are not met, a threshold test is not, in general, optimal.

In order to effect a likelihood ratio detector, it is necessary to obtain a valid estimate of the probability density functions of the data under $H_{1}$ and $H_{0}$. Under ideal conditions, in which both the clutter and targets are completely homogeneous, $s(t)=\exp \left(-\alpha_{1} t\right)$ under $H_{1}$ and $s(t)=\exp \left(-\alpha_{0} t\right)$ under $H_{0}$ ( $\alpha_{1}$ is thus representative of the target, while $\alpha_{0}$ is induced by the background, e.g., soil). In this case, $\alpha_{1}$ and $\alpha_{0}$ are assumed to be constant and known. Under these assumptions and assuming that the sensor is subject to additive Gaussian noise $n(k)$, with zero mean and known variance $\sigma^{2}$, then under $H_{1}$ the received signal, $r$, is

$$
r=\sum_{k=0}^{N-1}\left[\exp \left(-\alpha_{1}\left(t_{0}+k \Delta t\right)\right)+n(k)\right]
$$

and under $H_{0}$ the received signal is

$$
r=\sum_{k=0}^{N-1}\left[\exp \left(-\alpha_{0}\left(t_{0}+k \Delta t\right)\right)+n(k)\right] .
$$

(The variance $\sigma^{2}$ was computed from the data described in Section IV-A by calculating the variance in the responses obtained when the measurements were obtained at a single location multiple times prior to the beginning of the data collection.) Under these assumptions, the density functions follow a Gaussian distribution, or $p\left(r / H_{1}\right) \sim$ $N\left(K_{1}, N \sigma^{2}\right)$ and $p\left(r / H_{0}\right) \sim N\left(K_{0} \sim N \sigma^{2}\right)$, where $K_{1}=$ $\sum_{k=0}^{N-1} \exp \left(-\alpha_{1}\left(t_{0}+k \Delta t\right)\right)$ and $K_{0}=\sum_{k=0}^{N-1} \exp \left(-\alpha_{0}\left(t_{0}+\right.\right.$ $k \Delta t)$ ). When $K_{1}>K_{0}$, the threshold detector is in fact optimum.

2) Unknown Parameter Case: When the parameters $\alpha_{1}$ and $\alpha_{0}$ are not known exactly, or are known to vary spatially, the probability density distributions under the two hypotheses will not be as described above. Since targets and clutter are rarely homogeneous, we expect that these assumptions are not met in practice; this was confirmed upon inspection of the EM61 data from the DARPA study. In this case, the LRT becomes [6]

$$
\Lambda(r)=\frac{\int p\left(r / H_{1}, \alpha\right) p\left(\alpha_{1}\right) d \alpha_{1}}{\int p\left(r / H_{0}, \alpha_{0}\right) p\left(\alpha_{0}\right) d \alpha_{0}}
$$

where $p\left(\alpha_{1}\right)$ and $p\left(\alpha_{0}\right)$ are the probability density functions describing $\alpha_{1}$ and $\alpha_{0}$, respectively.

In the mine detection problem, it is usually possible to develop estimates of these probability density functions prior to initiating the detection process either by gathering data and estimating the density functions, or by employing a priori information regarding the metal content, and thus decay rates, of the targets in question. In this instance, the former approach was taken, both since a "calibration area" was provided in the data set (see Section IV-A for a description) and the statistical nature of the clutter process was expected to vary across the data collection sites. In order not to overfit the measured data, the parameters of either a Gaussian model (i.e., mean and 
variance) or a uniform model (i.e., upper and lower limits) were estimated from the data. This approach was followed for all of the sensors. In all cases, the performance did not vary significantly as a function of the statistical form of the model that was used.

To simplify the notation, let $s\left(\alpha_{1}\right)=\sum_{k=0}^{N-1} \exp \left(-\alpha_{1}\left(t_{0}+\right.\right.$ $k \Delta t))$ and $s\left(\alpha_{0}\right)=\sum_{k=0}^{N-1} \exp \left(-\alpha_{0}\left(t_{0}+k \Delta t\right)\right)$. For the case of additive white Gaussian noise, the LRT can be expressed as

$$
\Lambda(r)=\frac{\int_{\alpha_{1}^{L}}^{\alpha_{1}^{U}} \exp \left(-\frac{\left(r-s\left(\alpha_{1}\right)\right)^{2}}{2 N \sigma^{2}}\right) p\left(\alpha_{1}\right) d \alpha_{1}}{\int_{\alpha_{0}^{L}}^{\alpha_{0}^{U}} \exp \left(\frac{\left(r-s\left(\alpha_{0}\right)\right)^{2}}{2 N \sigma^{2}}\right) p\left(\alpha_{0}\right) d \alpha_{0}}
$$

where the limits of integration $\alpha_{1}^{U}, \alpha_{1}^{L}, \alpha_{0}^{U}$ and $\alpha_{0}^{L}$ are determined from the appropriate density functions and may be plus or minus infinity in some cases.

Although this integral can be calculated via Monte Carlo integration [7], we will prove that when the mean of the density function of $\alpha_{1}$ is less than the mean of the density function of $\alpha_{0}$ (i.e., responses to targets decay more slowly on the average than responses to the background or clutter), this likelihood ratio is a monotonic function of $r$, and therefore $r$ is a sufficient statistic [6]. Thus, if the likelihood ratio is a monotonic function of $r$, a threshold test of the received data is optimal, even when the parameters of the data are uncertain. To prove this relationship, we take the derivative of the likelihood ratio with respect to the received data $r$ and show that it is always greater than zero. Writing $\Lambda(r)$ as $\Lambda(r)=f(r) / g(r)$, we need to demonstrate that

$$
h(r)=\left(\frac{\partial}{\partial r} f(r)\right) g(r)-f(r)\left(\frac{\partial}{\partial r} g(r)\right)>0 .
$$

Taking the appropriate derivatives

$$
\begin{aligned}
h(r)= & \int_{\alpha_{1}^{L}}^{\alpha_{1}^{U}} \frac{-2\left(r-s\left(\alpha_{1}\right)\right)}{2 N \sigma^{2}} \exp \\
& \left(-\frac{\left(r-s\left(\alpha_{1}\right)\right)^{2}}{2 N \sigma^{2}}\right) p\left(\alpha_{1}\right) d \alpha_{1} \int_{\alpha_{0}^{L}}^{\alpha_{0}^{U}} \exp \\
& \left(-\frac{\left(r-s\left(\alpha_{0}\right)\right)^{2}}{2 N \sigma^{2}}\right) p\left(\alpha_{0}\right) d \alpha_{0} \\
& -\int_{\alpha_{0}^{L}}^{\alpha_{0}^{U}}-2\left(r-s\left(\alpha_{0}\right)\right) \\
2 N \sigma^{2} & \exp \\
& \left(-\frac{\left(r-s\left(\alpha_{0}\right)\right)^{2}}{2 N \sigma^{2}}\right) p\left(\alpha_{0}\right) d \alpha_{0} \int_{\alpha_{1}^{L}}^{\alpha_{1}^{U}} \exp \\
& \left(-\frac{\left(r-s\left(\alpha_{1}\right)\right)^{2}}{2 N \sigma^{2}}\right) p\left(\alpha_{1}\right) d \alpha_{1} .
\end{aligned}
$$

Simplifying, and combining the integrals

$$
\begin{aligned}
h(r)= & \frac{1}{N \sigma^{2}} \int_{\alpha_{1}^{L}}^{\alpha_{1}^{U}} \int_{\alpha_{0}^{L}}^{\alpha_{0}^{U}}\left(s\left(\alpha_{1}\right)-s\left(\alpha_{0}\right)\right) \exp \\
& \left(-\frac{\left(r-s\left(\alpha_{1}\right)\right)^{2}}{2 N \sigma^{2}}\right) \exp \left(-\frac{\left(r-s\left(\alpha_{0}\right)\right)^{2}}{2 N \sigma^{2}}\right) \\
& \cdot p\left(\alpha_{1}\right) p\left(\alpha_{0}\right) d \alpha_{1} d \alpha_{0} .
\end{aligned}
$$

Because probability density functions are always greater than or equal to zero, $h(r)$ is always greater than or equal to zero if the first term of the above equation $s\left(\alpha_{1}\right)-s\left(\alpha_{0}\right)$ is greater than zero. When $\alpha_{1}<\alpha_{0}$, the first term is positive and $h(r)>0$. If $\alpha_{1}$ is not always less than $\alpha_{0}$, but the mean of $p\left(\alpha_{1}\right)$ is less than the mean of $p\left(\alpha_{0}\right)$, the double integral can be divided into four areas of integration as

$$
\begin{aligned}
& \int_{\alpha_{0}^{L}}^{\alpha_{1}^{U}}\left(\int_{\alpha_{1}^{L}}^{\alpha_{0}^{L}}() d \alpha_{1}+\int_{\alpha_{0}^{L}}^{\alpha_{1}^{U}}() d \alpha_{1}\right) d \alpha_{0} \\
& \quad+\int_{\alpha_{1}^{U}}^{\alpha_{0}^{U}}\left(\int_{\alpha_{1}^{L}}^{\alpha_{0}^{L}}() d \alpha_{1}+\int_{\alpha_{0}^{L}}^{\alpha_{1}^{U}}() d \alpha_{1}\right) d \alpha_{0} .
\end{aligned}
$$

In the first, third, and fourth integrals, $\alpha_{1}<\alpha_{0}$, so these contribute only positive area to $h(r)$. For example, in the first integral, $\alpha_{1}^{L}<\alpha_{1}<\alpha_{0}^{L}$ and $\alpha_{0}^{L}<\alpha_{0}<\alpha_{1}^{U}$; thus, $\alpha_{1}<\alpha_{0}$. In the second integral, the fact that $\alpha_{1}$ and $\alpha_{0}$ are integrated over identical regions, coupled with the additive nature of and similarity in form of $s\left(\alpha_{1}\right)$ and $s\left(\alpha_{0}\right)$ results in a contribution of zero from this term since

$$
\begin{aligned}
\int_{\alpha_{0}^{L}}^{\alpha_{1}^{U}} \int_{\alpha_{0}^{L}}^{\alpha_{1}^{U}} & {\left[\sum_{k=0}^{N-1} \exp \left(-\alpha_{1}\left(t_{0}+k \Delta t\right)\right)\right.} \\
& \left.-\sum_{k=0}^{N-1} \exp \left(-\alpha_{0}\left(t_{0}+k \Delta t\right)\right)\right] d \alpha_{1} d \alpha_{0} \\
=\int_{\alpha_{0}^{L}}^{\alpha_{1}^{U}} & {\left[\sum_{k=0}^{N-1} \int_{\alpha_{0}^{L}}^{\alpha_{1}^{U}} \exp \left(-\alpha_{1}\left(t_{0}+k \Delta t\right)\right) d \alpha_{0}\right.} \\
& \left.-\sum_{k=0}^{N-1} \int_{\alpha_{0}^{L}}^{\alpha_{1}^{U}} \exp \left(-\alpha_{0}\left(t_{0}+k \Delta t\right)\right) d \alpha_{0}\right] d \alpha_{1}
\end{aligned}
$$$$
=0 \text {. }
$$

Therefore, $h(r)>0$ for the previously noted assumptions on the parameters involved.

This analysis has shown that, for integrated time-domain sensors, such as the EM61 operating under the assumption of Gaussian sensor noise with known statistics, the optimal processor for the detection of targets is a simple threshold statistic. This result follows directly from the likelihood ratio when the target and clutter responses are known exactly. However, even under conditions of uncertainty, as long as the rate of decay of target signatures is slower on average than that of clutter, the likelihood ratio is a monotonic function of the integrated response, and thus the optimal processor is still a threshold test. It will be shown in the next section, however, that, if the entire signal is available for processing, the optimal processor is not a threshold test performed on the integrated data values.

\section{B. Time-Domain Sensors}

In addition to incorporating a model of the received waveform into the detector formulation, we exploit the fact that an EMI device is effectively a "change detector," as long as the size of the objects of interest are small with respect to the spacing of the response measurements. This approach implicitly incorporates the assumption that the background 
clutter is spatially correlated. For simplicity, it is assumed that, when no target is present, the statistics of the responses at two adjacent points are the same. However, if a small metal and/or permeable target is present, the response at the two locations will necessarily be different. The target must be small with respect to the spacing of the response measurements to ensure that target responses are not observed at sequential measurement locations.

By taking this approach, it is not necessary to specify the target response exactly, as would be the case for a matched filter [6]. This approach also avoids limiting the detector to searching for a particular decay rate, which corresponds to looking for a particular type of metal. Instead, this approach allows the uncertainty in the ground surface, composition of the mine, and its placement in the surrounding environment to be incorporated into the design of the processor.

Let $r_{x, y}(k)$ be the normalized, sampled, received signal at location $\{x, y\}$ on a site, where

$$
r_{x, y}(k)=s_{x, y}(k)+n_{x, y}(k) .
$$

Again, $n_{x, y}(k)$ is assumed to be a white Gaussian noise process with known statistics associated with the sensor, and as before

$$
s_{x, y}(k)=\exp \left(-\alpha_{x, y}\left(t_{0}+k \Delta t\right)\right)
$$

Note that under the no-target, or null hypothesis $\left(H_{0}\right), \alpha_{x, y}$ is associated with the response of the ground, or a clutter object, whereas under the target hypothesis, $H_{1}, \alpha_{x, y}$ is determined by the target response. If $\alpha_{x, y}=\alpha_{1}$ is a single, known constant for targets, and a different, known constant for clutter $\left(\alpha_{0}\right)$, the optimum test is a matched filter [6], or correlation receiver, in which the received signal is correlated with the difference between a decaying exponential due to a target and that due to clutter, or

$$
\begin{aligned}
\Lambda\left(\mathbf{r}_{x, y}\right)= & \sum_{k=0}^{N-1} r_{x, y}(k)\left[\exp \left(-\alpha_{1}\left(t_{0}+k \Delta t\right)\right)\right. \\
& \left.-\exp \left(-\alpha_{0}\left(t_{0}+k \Delta t\right)\right)\right] .
\end{aligned}
$$

It is important to note that, under these assumptions, the optimal processing approach is a weighted sum of the received data, and not an unweighted sum, such as that performed by integrated EMI sensors. However, modeling and experimental results, such as those obtained in the DARPA experiment, indicate that the decay rates across targets and clutter are quite variable. Therefore, the matched filter approach is not necessarily optimal.

An alternate approach can be obtained by assuming that the operator travels linearly in the $y$ direction, targets are small with respect to the data sampling rate, and the terrain characteristics are slowly varying in space. Note that, although this approach is not appropriate without modification for targets that extend across multiple spatial locations, it is useful to help screen out clutter items that are extended in space. Given these assumptions, under $H_{0}$

$$
s_{x, y}(k)=s_{x, y-1}(k)
$$

or equivalently

$$
\alpha_{x, y}(k)=\alpha_{x, y-1}(k) .
$$

Under the target hypothesis, $H_{1}, s_{x, y-1}(k)$ and $s_{x, y-1}(k)$ are not identical, such that

$$
\alpha_{x, y}(k) \neq \alpha_{x, y-1}(k) .
$$

Based on these preliminaries, the nature of the received signal $r_{x, y}(k)$ at locations $\{x, y\}$ and $\{x, y-1\}$ may be determined. Under $H_{0}$

$$
\begin{aligned}
r_{x, y}(k) & =\exp \left(-\alpha_{0}\left(t_{0}+k \Delta t\right)\right)+n_{x, y} \\
r_{x, y-1}(k) & =\exp \left(-\alpha_{0}\left(t_{0}+k \Delta t\right)\right)+n_{x, y-1}
\end{aligned}
$$

where $n_{x, y}$ is assumed to be an independent, identically distributed Gaussian random process with zero mean and known variance $\sigma^{2}$ (estimated as described in the previous section). Under $H_{1}$

$$
\begin{aligned}
r_{x, y}(k) & =\exp \left(-\alpha_{1}\left(t_{0}+k \Delta t\right)\right)+n_{x, y} \\
r_{x, y-1}(k) & =\exp \left(-\alpha_{0}\left(t_{0}+k \Delta t\right)\right)+n_{x, y-1} .
\end{aligned}
$$

Again, the LRT for the received data vector $\mathbf{r}$ is defined as

$$
\Lambda(\mathbf{r})=\frac{p\left(\mathbf{r} / H_{1}\right)}{p\left(\mathbf{r} / H_{0}\right)}
$$

where $p\left(\mathbf{r} / H_{1}\right)$ and $p\left(\mathbf{r} / H_{0}\right)$ are the probability density functions of the received data vector under $H_{1}$ and $H_{0}$, respectively. As before, when $\Lambda(\mathbf{r})>\delta, H_{1}$ is chosen, whereas when $\Lambda(\mathbf{r})<\delta, H_{0}$ is chosen.

Since the distribution of the received signal $\mathbf{r}$ is dependent on the parameters $\alpha_{1}$ and $\alpha_{0}$ under $H_{1}$, and on $\alpha_{0}$ under $H_{0}$, and these parameters are unknown, if we let $s_{\alpha_{1}}(k)=$ $\exp \left(-\alpha_{1}\left(t_{0}+k \Delta t\right)\right)$ and $s_{\alpha_{0}}(k)=\exp \left(-\alpha_{0}\left(t_{0}+k \Delta t\right)\right)$, the LRT becomes as in (23), shown at the bottom of the page, where $p\left(\alpha_{0}\right)$ and $p\left(\alpha_{1}\right)$ are the probability density functions associated with the decay times $\alpha_{1}$ and $\alpha_{0}$. In this case, Monte Carlo integration [7] is utilized to calculate the decision statistic specified above.

This decision statistic can be computationally expensive since Monte Carlo integration of the integrals can, in some cases, take many iterations to converge. Therefore, the suboptimal generalized likelihood ratio test (GLRT) has also been formulated [6]. This formulation avoids the evaluation

$$
\frac{\iint\left(\prod_{k} \exp \left(-\frac{\left(r_{x, y-1}(k)-s_{\alpha_{0}}(k)\right)^{2}}{2 \sigma^{2}}-\frac{\left(r_{x, y}(k)-s_{\alpha_{1}}(k)\right)^{2}}{2 \sigma^{2}}\right)\right) p\left(\alpha_{0}\right) p\left(\alpha_{1}\right) d \alpha_{0} d \alpha_{1}}{\int\left(\prod_{k} \exp \left(-\frac{\left(r_{x, y-1}(k)-s_{\alpha_{0}}(k)\right)^{2}}{2 \sigma^{2}}-\frac{\left(r_{x, y}(k)-s_{\alpha_{0}}(k)\right)^{2}}{2 \sigma^{2}}\right)\right) p\left(\alpha_{0}\right) d \alpha_{0}}
$$


of the multidimensional integration and results in a simplified detector. In the GLRT formulation, the maximum likelihood estimate for each of the unknown parameters (in this case, $\alpha_{1}$ and $\alpha_{0}$ ) is used in the formulation of the likelihood ratio. For this problem, the GLRT has the form

$$
\Lambda(\mathbf{r})=\frac{\prod_{k} \exp \left(-\frac{\left(r_{x, y}(k)-\hat{s}_{x, y}\left(k, \alpha_{1}\right)\right)^{2}}{2 \sigma^{2}}\right)}{\prod_{k} \exp \left(-\frac{\left(r_{x, y}(k)-\hat{s}_{x, y}\left(k, \alpha_{0}\right)\right)^{2}}{2 \sigma^{2}}\right)} .
$$

Here, $\hat{s}_{x, y}\left(k, \alpha_{1}\right)$ and $\hat{s}_{x, y}\left(k, \alpha_{0}\right)$ are the maximum likelihood estimators [6] for $s_{x, y}\left(k, \alpha_{1}\right)$ and $s_{x, y}\left(k, \alpha_{0}\right)$, and they can be estimated from the appropriate $r_{x, y}(k)$. In this case, the likelihood estimator for the mean is simply the sample mean [6] and this estimator in (24) is applied to the data obtained in the calibration area (see Section IV-A).

In order to determine whether the change detector formulation provides an improvement in performance over a pixel-by-pixel Bayesian approach, the performance using the likelihood ratio for the pixel-by-pixel case was also evaluated. In this case, Monte Carlo integration was used to evaluate

$$
\Lambda(r)=\frac{\int \prod_{k} \exp \left(-\frac{\left(r_{x, y}(k)-s_{x, y}\left(k, \alpha_{1}\right)\right)^{2}}{2 \sigma^{2}}\right) p\left(\alpha_{1}\right) d \alpha_{1}}{\int \prod_{k} \exp \left(-\frac{\left(r_{x, y}(k)-s_{x, y}\left(k, \alpha_{0}\right)\right)^{2}}{2 \sigma^{2}}\right) p\left(\alpha_{0}\right) d \alpha_{0}} .
$$

The GLRT formulation for the pixel-by-pixel analysis is equivalent to that shown in (24). In all cases, parameters of the a priori density functions, such as $p\left(\alpha_{1}\right)$, were estimated from the data measured in the calibration area (see Sections IV-A and III-A).

\section{Wideband Frequency-Domain Sensors}

In order to effect a likelihood ratio detector for each of the components of a frequency-domain sensor (in-phase and quadrature measured for each frequency), as well as for the entire data set, it is again necessary to obtain a valid estimate of the probability density functions of the data under $H_{1}$ and $H_{0}$. Based on the data that were gathered with the GEM3 system during the DARPA experiment, we will restrict the development to the case in which data are measured at two frequencies, resulting in four components. If each of the recorded components is a deterministic, known quantity for all targets and types of clutter, and is subject to additive, zeromean Gaussian noise, denoted $n$, under $H_{1}$, the $i$ th received component can be modeled as

$$
r_{i}=A_{1, i}+n \text {. }
$$

In the case of the GEM-3, $i=1$ denotes the in-phase component at $4050 \mathrm{~Hz}, i=2$ denotes the quadrature component at the same frequency, etc. Under $H_{0}$, the $i$ th received component can be modeled as

$$
r_{i}=A_{0, i}+n
$$

where $A_{1, i}>A_{0, i}$. In this idealized case, the threshold detector is in fact optimal.

However, in the case in which the parameters $A_{1, i}$ and $A_{0, i}$ are not known exactly, or are known to vary spatially, the probability density distributions will be more complicated in general than described above. Since targets and clutter are rarely homogeneous, we expect that these assumptions are in general not valid (confirmed through data collected with the GEM-3 during the DARPA experiment). To mitigate this problem, two approaches have been taken. In the first, the density functions are estimated from the collected data, using both parametric [8] and nonparametric [9] approaches (similar to the approach used for the time-domain and integrated timedomain sensors). These density functions are then used to form the likelihood ratio test. A second approach, which does not rely so directly on the data, invokes the Gaussian assumption, with parameters assumed unknown. In this case, the likelihood ratio test becomes

$$
\Lambda\left(r_{i}\right)=\frac{\int p\left(r_{i} /\left(H_{1}, A_{1, i}\right)\right) p\left(A_{1, i}\right) d A_{1, i}}{\int p\left(r_{i} /\left(H_{0}, A_{0, i}\right)\right) p\left(A_{0, i}\right) d A_{0, i}}
$$

where $p\left(A_{1, i}\right)$ and $p\left(A_{0, i}\right)$ are the probability density functions for $A_{1, i}$ and $A_{0, i}$, respectively. Again, the parameters of these density functions were estimated from data taken in the calibration area for each site (see Section IV-A). More specifically

$$
\Lambda\left(r_{i}\right)=\frac{\int \exp \left(-\frac{\left(r_{i}-A_{1, i}\right)^{2}}{2 \sigma^{2}}\right) p\left(A_{1, i}\right) d A_{1, i}}{\int \exp \left(-\frac{\left(r_{i}-A_{0, i}\right)^{2}}{2 \sigma^{2}}\right) p\left(A_{0, i}\right) d A_{0, i}} .
$$

Monte Carlo integration is used to evaluate the integrals in the above equation.

The same two approaches were used to develop a detector for the full data set (in-phase and quadrature at 4050 and $12270 \mathrm{~Hz}$ ). In the first approach, the multidimensional density functions are estimated from the collected data and these density functions are then used to form the likelihood ratio test. In the second approach, the Gaussian assumption is used, but the parameters of the distribution are assumed to be unknown.

\section{Threshold Detectors and Other Suboptimal Detectors}

In order to address whether the complexity of the optimal receiver results in significant performance improvements, and to compare the results of the model-based processor to those of other processors, several other simplified detection strategies are investigated. In these approaches, the physics of the target and ground responses are utilized to realize ad hoc detection strategies [10]-[12].

When the clutter and target data follow Gaussian distributions with identical variances, but different means, the LRT reduces to the standard threshold test for unidimensional data, or to the matched filter in the multidimensional case. A threshold test is commonly applied to EMI data, and it thus was evaluated for the EM61 data, along with each of the individual 


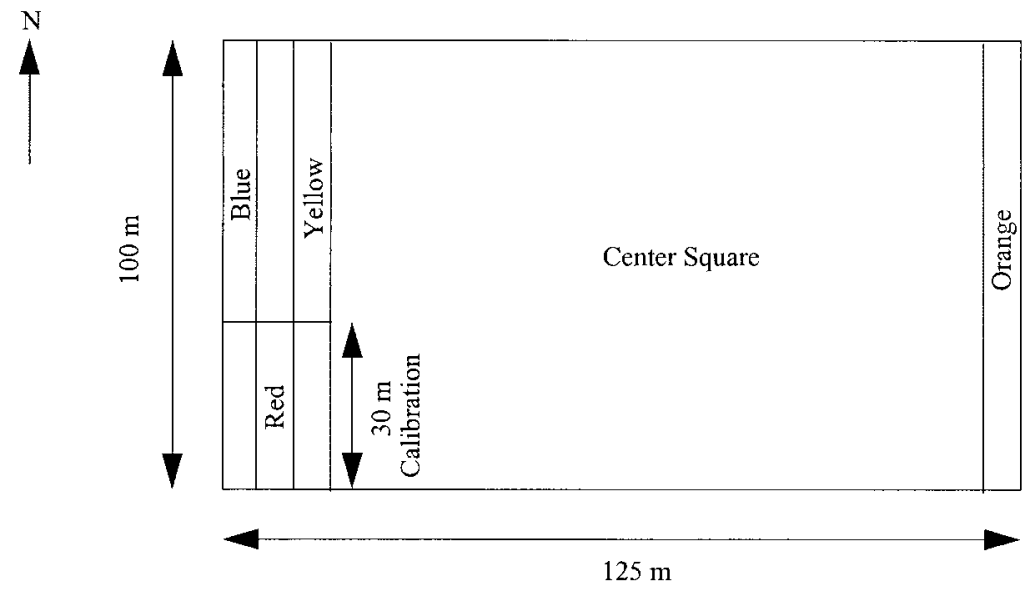

Fig. 1. Typical site layout from DARPA backgrounds clutter data collection experiment.

components of the GEM-3. The detection performance of a matched filter was also analyzed for the EM61-3D. For the matched filter, the received signal was correlated with the difference between a "known" signal (the "known" signal was extracted from the data of targets that were emplaced in the site) and the average clutter signal. Similar results to those described below were obtained for all "known" target signals.

Under the hypothesis that the ground is less conductive than potential targets, the rate of decay of the clutter signatures will be faster than that of the targets (this neglects anthropic clutter with significant metal content). Several detectors may be formulated to exploit this observation. One such detector is an energy detector, which was applied to the EM61-3D and the GEM-3 data. In addition, for the EM61-3D, a decay rate estimator was formulated. In the results presented here, a least-squares estimate of the rate of decay applied [7].

\section{EXPERIMENTAL DETAILS}

\section{A. Data}

The objective of the background clutter data collection experiment [1] was to collect data to aid in the understanding of the effects of clutter on system performance. During the course of the experiment, data were collected using four types of sensors: ground penetrating radar (GPR), EMI, magnetometer, and infrared (IR). Data were collected at four sites, distributed over two locations (Fort Carson, CO, and Fort A. P. Hill, VA). The locations represented three different soil types, along with substantial variability in man-made contamination (anthropic clutter). Three of the four sites had a small amount of anthropic clutter, while at one of the sites (Fort A. P. Hill) the amount of such was substantial. In addition to the indigenous anthropic clutter, a few synthetic calibration targets, unexploded ordnance (UXO) items, and land mines were emplaced on each site.

A typical layout for a test site is illustrated in Fig. 1. The site measured $125 \times 100 \mathrm{~m}$ and consisted of three areas: a center square, side bars, and a calibration area. The center square, measuring $100 \times 100 \mathrm{~m}$, was intended primarily for clutter characterization, although 15 calibration targets were placed there to aid equipment calibration and location registration.
Replicas of the calibration targets, along with synthetic UXO items and land mines were emplaced in the red, blue, yellow, and orange side bars on the site. A complete description of the targets and their deployment can be found in [1]. In general, the red lane consisted of copies of the registration targets, other targets for calibration, and system-stressing targets. The blue side bar contained UXO and demagnetized spheres, the yellow side bar contained mines, IR calibration sources, and dielectric targets. The orange side bar consisted of a combination of these and other targets. The total area of the blue yellow and orange side bars was $1300 \mathrm{~m}^{2}$. The calibration area consisted of a 30 $\times 15 \mathrm{~m}$ portion of the red, blue, and yellow side bars. The locations and descriptions of the targets in the calibration area (14 of which could be seen by EMI systems) and the center square (ten of which could be seen by EMI systems) were released to the public, the remaining locations (blue, yellow, and orange side bars) were not disclosed. In the analyses that are described below, only data from the calibration areas and the center square are processed to evaluate performance of the various detectors.

\section{B. Sensors}

In this paper, we consider data from three classes of EMI sensors: sensors that integrate time-domain information to provide a single data point (standard metal detector), those that provide a sampled portion of the time-domain waveform, and those that operate at several discrete frequencies.

The first system, the Geonics EM61, consists of a singlechannel pulsed induction system with a $0.5-\mathrm{m}$ transmitter coil positioned approximately $0.3 \mathrm{~m}$ above the ground. Data are received in both the transmitter coil and a second receiver coil that is located $0.4 \mathrm{~m}$ above the transmitter. The system operates at a center frequency of $75 \mathrm{~Hz}$; the received signal is integrated from 0.18 to $0.87 \mathrm{~ms}$ after each transmit pulse, and the result, a single data point, is stored for later processing. Data were collected along survey lines spaced $0.5 \mathrm{~m}$ in the easterly direction and $0.2 \mathrm{~m}$ on the average in the northerly direction.

The second sensor, the Geonics EM61-3D, is a prototype three-component time-domain induction system having a 1- 


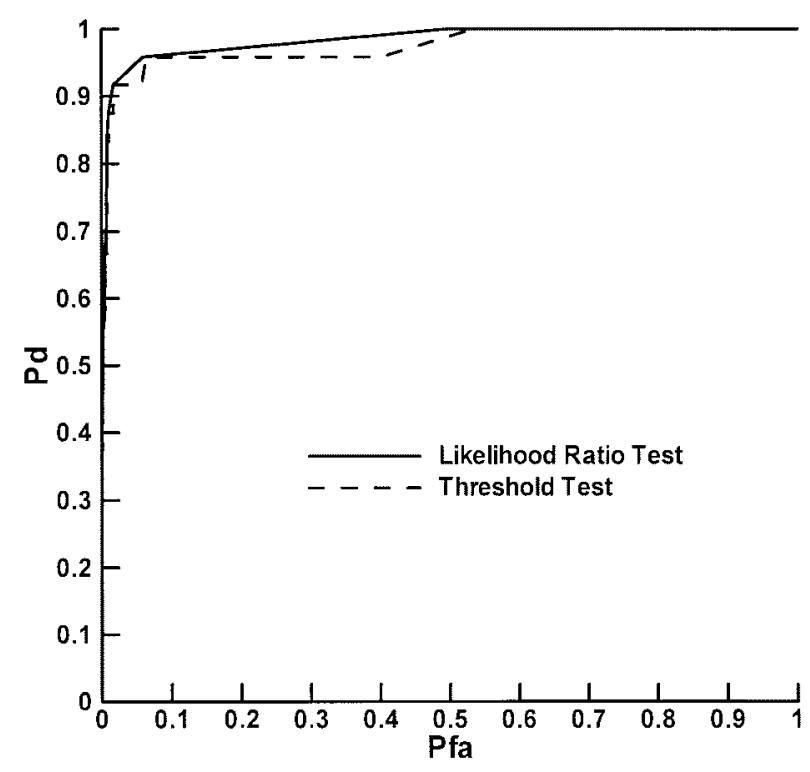

Fig. 2. Comparison of threshold (dashed line) and likelihood ratio test (solid line) performance-EM61, Turkey Creek site.

$\mathrm{m}$ square transmitter coil and three orthogonal $0.5-\mathrm{m}$ receiver coils positioned approximately $0.3 \mathrm{~m}$ above the ground. The system operates at a center frequency of $7.5 \mathrm{~Hz}$. Sensor output is measured and recorded at 20 geometrically spaced time gates, spanning a time range from 32 to $320 \mu \mathrm{s}$. Data were collected along survey lines spaced $1 \mathrm{~m}$ apart in the easterly direction and at a rate of 3 samples per second, or approximately $0.2 \mathrm{~m}$ in the northerly direction.

The third system that was analyzed was Geophex's prototype GEM-3 sensor [13]. The GEM-3 is a monostatic, multifrequency EMI instrument. The GEM-3 can function as a time- or frequency-domain sensor; however, for the DARPA data collection project, the frequency-domain capabilities were used exclusively. Data acquisition parameters for all four test sites included a $10-\mathrm{Hz}$ sampling rate and two transmit frequencies, 4050 and $12270 \mathrm{~Hz}$. The data used for detection in the subsequent analysis consist of in-phase and quadrature readings at each transmit frequency. Data were collected along survey lines spaced $0.5 \mathrm{~m}$ apart in the easterly direction and $0.17 \mathrm{~m}$ on the average in the northerly direction.

\section{RESUlTS}

In this section, the results of the different detection strategies are discussed. The raw data were analyzed by dividing each site into an appropriate grid based on the sampling rate of each of the sensors, and then averaging all signals recorded within each grid point. In the interest of space, data from only one site is presented in the figures for each sensor, although variability in performance across sites is discussed.

\section{A. Integrated Time-Domain Sensor: EM61}

As was expected based on the analysis presented in Section III-A, Fig. 2 indicates no difference between the performance achieved using the Bayesian processor and a standard thresholding technique. This results because the likelihood ratio is a

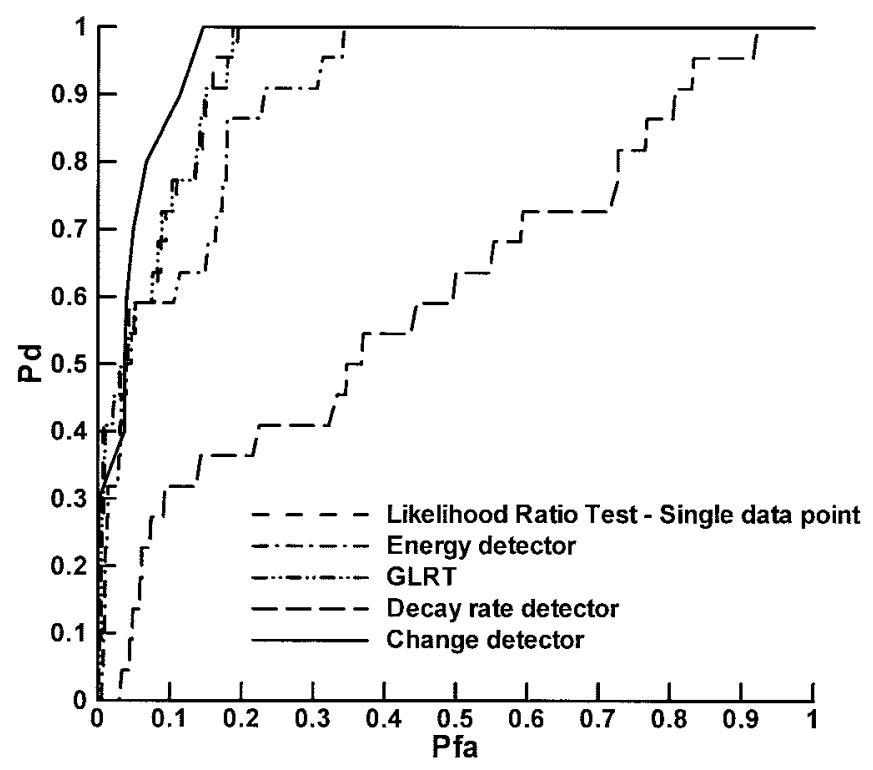

Fig. 3. Comparison of detector performance-EM61-3D, firing point 20 site. Solid line $=$ Bayesian change detector performance, dashed line $=$ Bayesian pixel-by-pixel detector, dotted line = GLRT performance, dashed-dotted line $=$ energy detector, long-dashed line $=$ decay rate detector.

monotonic function of the data (Section II-A) when the data are the integrated time-domain signals. Again, it should be emphasized that this is not the case when samples of the raw time-domain signal are available for processing.

\section{B. Time-Domain Sensor: EM61-3D}

The superiority of the single pixel Bayesian processor, as compared to standard thresholding and various ad hoc techniques, is demonstrated for the EM61-3D in Fig. 3. The performance of the GLRT processor is approximately equivalent to that of the single-pixel Bayesian formulation. It is interesting to note that the energy detector is superior to the ad hoc processors based on the decay rate and the matched filter (not shown since the performance was almost identical to that of the decay-rate processor); this is likely a result of the significant variability in target and clutter signatures, not taken into account by either of the ad hoc processors. In addition, the Bayesian change detector formulation provides improved performance over the pixel-by-pixel formulation. Absolute performance of the different approaches varies across sites, and it is poorest at Firing Point 20, Ft. A. P. Hill, VA, known to be the most cluttered site. The detection rate for the Bayesian change detector formulation is larger than that of more standard approaches by a factor of between 1.4 and 2 at a fixed false alarm rate of $5 \%$, depending on the site.

\section{Wideband Frequency-Domain Sensor: GEM-3}

Fig. 4 illustrates the improvement in performance of the Bayesian approach relative to standard threshold techniques for the individual components measured by the GEM-3. Substantial improvements are observed for all components except the $12270 \mathrm{~Hz}$ in-phase component, for which the performance of the two approaches is equivalent. In some cases, detection performance at a false alarm rate of $5 \%$ can be improved by 


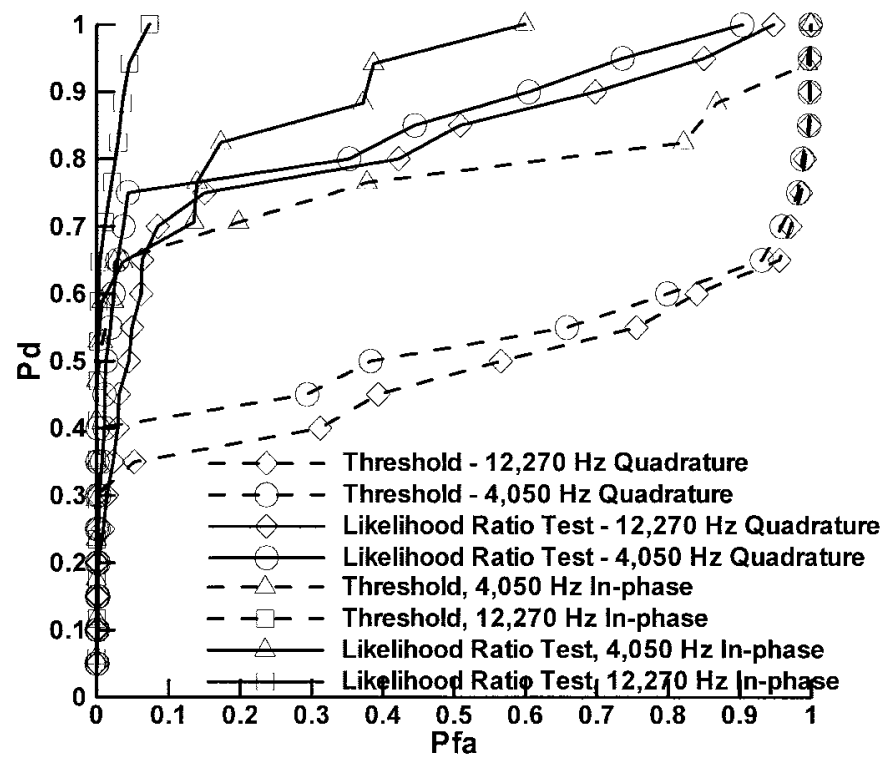

Fig. 4. Comparison of detection performance-single components of GEM-3, Firing Point 22 site. Dashed lines indicate detection performance for threshold tests, solid lines indicate Bayesian detector performance. Triangles $=4050 \mathrm{~Hz}$ in-phase data, circles $=4050 \mathrm{~Hz}$ quadrature data, squares $=12270 \mathrm{~Hz}$ in-phase data, diamonds $=12270 \mathrm{~Hz}$ quadrature data.

a factor of two to three. Similar performance improvements were observed across all four sites, with some variability in absolute level of performance. In all cases, the $12270 \mathrm{~Hz}$ data results in the best detection performance, regardless of the site. It is possible that the improved detection at $12270 \mathrm{~Hz}$ (over that at $4050 \mathrm{~Hz}$ ) is a result of differences in skin-depth effects between target and clutter that are only observable at higher frequencies. This result suggests that careful selection of the transmitting frequencies [14], based on the physics of the underlying process, may allow additional improvements in detection performance.

Fig. 5 illustrates the improvement in performance obtained when the entire data set is processed using the Bayesian approach, as compared to the best performance achievable with a single component (dashed line, $12270 \mathrm{~Hz}$, in-phase). The fused data set provides better detection performance across all sites than that achievable with a single component.

\section{CONCLUSIONS}

We have presented results which indicate that a rigorous, Bayesian signal detection theoretic analysis can be used to improve detectability of a set of targets, including land mines and some small UXO objects using EMI sensors over standard thresholding techniques. A suite of EMI sensors was evaluated, including those that integrate time-domain information to provide a single data point (standard metal detector), those that provide a sampled portion of the time-domain waveform, and those that operate at several discrete frequencies.

Theoretical analyses of the statistical nature of the signal obtained from standard metal detectors indicated that this signal was in fact monotonic with the likelihood ratio. Thus, a threshold detector is the optimal processor for a sensor that integrates the time-domain waveform to generate a single data

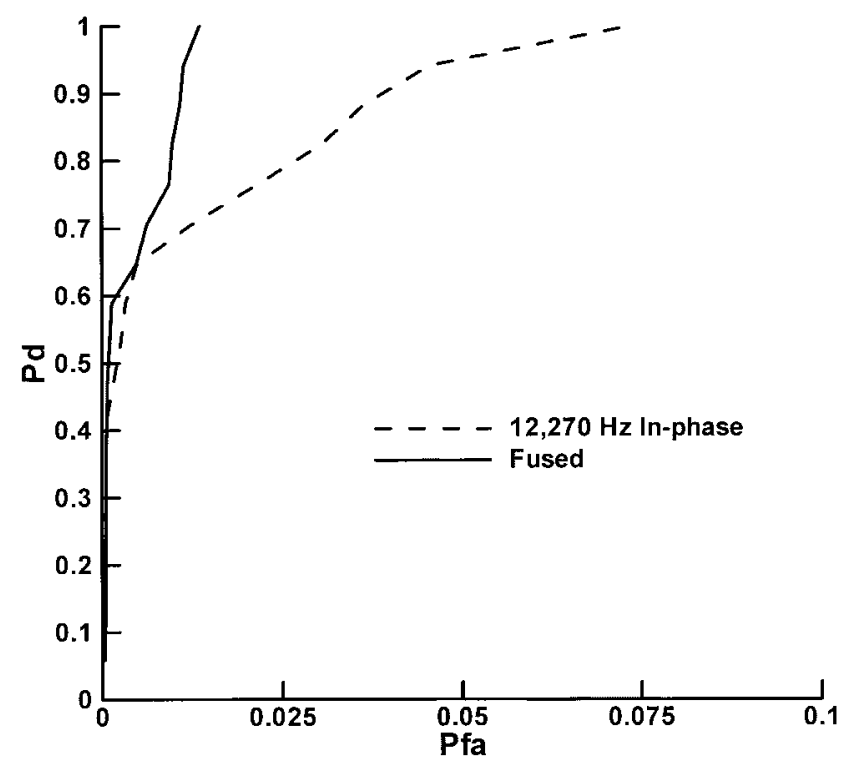

Fig. 5. Comparison of detector performance-fused (solid line) versus single component (dashed line, $12270 \mathrm{~Hz}$ in-phase) of GEM-3, Firing Point 22 site.

point. Using data obtained from the DARPA experiment, the performance of the likelihood ratio processor and a threshold detector were evaluated and shown to be equivalent. This indicated that the assumptions used to derive the LRT and its equivalence to a threshold test were valid, at least for the data in question.

When a sampled version of the induced time-domain response from the target is available for processing, further theoretical analysis indicated that an integration of the timedomain waveform is not the optimal form of processing. In this case, the optimal processor was implemented and its performance was compared not only to the performance of the threshold test, but to several ad hoc processors that have been suggested in the literature [11], [12]. The results of this analysis indicated that a Bayesian detector operating on the data exceeded that of either the threshold detector or the ad hoc strategies tested. In addition, a Bayesian approach developed using the notion that such sensors are effectively change detectors provided an additional improvement in detection performance.

Similar results were obtained for a Bayesian detector designed to process the measured frequency response from a target. The form of the detector did not reduce to a threshold test, and in all but one case, in which the performance of the two detectors was equivalent, the performance of the Bayesian detector far exceeded that of the threshold test. In addition, a detector designed to process all of the frequency data concurrently performed significantly better than the detectors designed to operate on the single-frequency data. The results from this analysis indicated that detection performance is a function of frequency and that careful selection of operating frequencies may result in further improvements in detection performance [14].

In the development of the likelihood ratios, probability density functions for the data, and for the parameters upon which the signals depended, were required. In general, these 
density functions were derived from the data that was acquired by each sensor in a calibration area, in which the location of the targets was known and the background, or clutter, was typical to that contained in the remainder of the test site. To avoid "overfitting" the data, parameters of two general classes of density functions, a Gaussian and a uniform, were estimated and the performance achieved using the different density functions was compared to evaluate the robustness of the methodology. The performance of these systems was only slightly affected by the selection of the density function, which is similar to results obtained in other studies [14]. It was necessary, however, to estimate these density functions separately for each site, particularly for the clutter statistics, since these statistics varied substantially across sites. Using an estimate of the density functions based on one of the sites at a different site usually degraded the performance substantially.

Although target responses to EMI systems have been studied both experimentally and theoretically [2]-[5], [10], it is only recently that multichannel time- and frequency-domain sensors have become available so that the Bayesian approach to detector design can be exploited. The DARPA experiment enabled a more complete characterization of statistics, thus aiding in the design of such detectors. We have shown that the approach provides improved detection performance over standard detection technique. In addition, the results presented here indicate that detection performance is improved if the entire time-domain signal, or multifrequency data are utilized since this allows exploitation of the phenomenology embedded in the signatures. The theoretical development of the statistically based algorithm, along with the proof of concept application of the detector formulation to the DARPA data, combined to yield encouraging progress toward the problem of mine detection.

\section{ACKNOWLEDGMENT}

The authors would like to thank Dr. T. Altshuler, V. George, and Dr. R. Dugan for many helpful discussions pertaining to this work. They would also like to thank the four anonymous reviewers for their helpful comments.

\section{REFERENCES}

[1] V. George, T. Altshuler, A. Andrews, J. Nicoll, E. Cespedes, D. Butler, T. Broach, and R. Mehta, "Background data collection plan," DARPA/Defense Science Office, Dec. 1996

[2] C. E. Baum, "Low-frequency near-field magnetic scattering from highly, but not perfectly, conducting bodies," Phillips Lab. Interaction Note 499, Nov. 1993.

[3] G. D. Sower and Steven P. Cave, "Detection and identification of mines from natural magnetic and electromagnetic resonances," in Proc. SPIE, Orlando, FL, Apr. 1995, vol. 2496.

[4] Y. Das, J. E. McFee, and R. H. Cherry, "Time-domain response of a sphere in the field of a coil: Theory and experiment," IEEE Trans. Geosci. Remote Sensing, vol. GE-22, pp. 360-367, July 1984.
[5] Y. Das and J. E. McFee, "A simple analysis of the electromagnetic response of buried conducting objects," IEEE Trans. Geosci. Remote Sensing, vol. 28, pp. 278-288, May 1990.

[6] H. L. Van Trees, Detection, Estimation, and Modulation Theory. New York: Wiley, 1968.

[7] J. O. Berger, Statistical Decision Theory. Berlin, Germany: SpringerVerlag, 1980

[8] B. W. Silverman, Density Estimation for Statistics and Data Analysis. New York: Chapman \& Hall, 1986.

[9] G. Roussas, Nonparametric Functional Estimation and Related Topics. New York: Kluwer, 1990.

[10] Y. Das, J. E. McFee, J. Toew, and G. C. Stuart, "Analysis of an electromagnetic induction detector for real-time location of buried objects," IEEE Trans. Geosci. Remote Sensing, vol. 29, pp. 343-344, Mar. 1991.

[11] A. C. Dubey, I. Cindrich, J. M. Ralston, and K. Rigano, Eds., "Detection technologies for mines and minelike targets," in Proc. SPIE, Orlando, FL, Apr. 17-21, 1995, vol. 2496.

[12] A. C. Dubey and R. L. Barnard, Eds., "Detection and remediation technologies for mines and minelike targets H," in Proc. SPIE, Orlando, FL, Apr. 21-24, 1997, vol. 3079.

[13] I. J. Won, D. A. Keiswetter, and D. R. Hanson, "GEM-3: A monostatic broadband electromagnetic induction sensor," J. Environ. Eng. Geophys., vol. 2, pp. 53-64, Mar. 1997.

[14] N. Geng, P. Garber, L. Collins, L. Carin, D. Hansen, D. Keiswetter, and I. J. Won, "Wideband electromagnetic induction for metal-target identification: Theory, measurement and signal processing," Tech. Rep. Duke Univ., Durham, NC, Sept. 1997.

Leslie Collins (M'96) was born in 1963 in Raleigh, NC. She received the B.S.E.E. degree in 1985 from the University of Kentucky, Lexington, and the M.S.E.E. degree in 1986 and the Ph.D. degree in electrical engineering in 1995, both from The University of Michigan, Ann Arbor.

She was a Senior Engineer at the Westinghouse Research and Development Center, Pittsburgh, PA, from 1986 to 1990. In 1995, she became an Assistant Professor in the Electrical and Computer Engineering Department at Duke University, Durham, NC. Her current research interests include incorporating physics-based models into statistical signal processing algorithms, and she is pursuing applications in demining as well as enhancing speech understanding by hearing impaired individuals.

Dr. Collins is a member of the Tau Beta Pi, Eta Kappa Nu, and Sigma $\mathrm{Xi}$ honor societies.

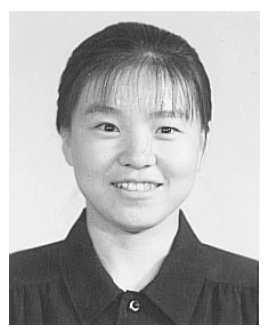

Ping Gao (S'97) received the B.S. and M.E. degrees (honors) in electrical engineering from Beijing University of Posts and Telecommunications, Beijing, China, in 1993 and 1996, respectively, and the M.S.E.E. degree from Duke University, Durham, $\mathrm{NC}$, in 1997. She is currently pursuing the Ph.D. degree in the Electrical and Computer Engineering Department at Duke University.

She has worked as a Research Assistant at Duke University since 1997 . Her main research interests include signal detection and estimation theory, statistical signal modeling and processing, and applied electromagnetics.

Lawrence Carin (S'85-M'89-SM'96), for a photograph and biography, see p. 359 of the January 1999 issue of this Transactions. 